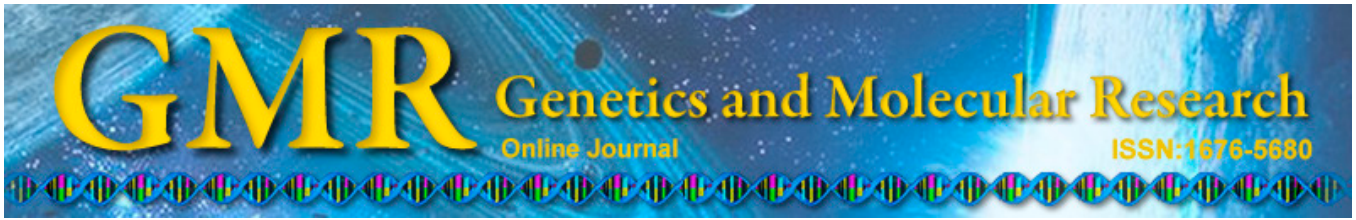

\title{
Prediction of protein-protein interactions using chaos game representation and wavelet transform via the random forest algorithm
}

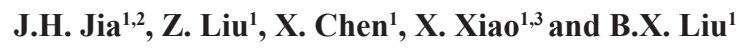 \\ ${ }^{1}$ School of Information Engineering, Jingdezhen Ceramic Institute, \\ Jingdezhen, China \\ ${ }^{2}$ School of Computer Science, University of Birmingham, Edgbaston, \\ Birmingham, UK \\ ${ }^{3}$ Gordon Life Science Institute, Boston, MA, USA \\ Corresponding author: J.H. Jia \\ E-mail: jjh163yx@163.com
}

Genet. Mol. Res. 14 (4): 11791-11805 (2015)

Received January 23, 2015

Accepted May 15, 2015

Published October 2, 2015

DOI http://dx.doi.org/10.4238/2015.October.2.13

\begin{abstract}
Studying the network of protein-protein interactions (PPIs) will provide valuable insights into the inner workings of cells. It is vitally important to develop an automated, high-throughput tool that efficiently predicts protein-protein interactions. This study proposes a new model for PPI prediction based on the concept of chaos game representation and the wavelet transform, which means that a considerable amount of sequence-order effects can be incorporated into a set of discrete numbers. The advantage of using chaos game representation and the wavelet transform to formulate the protein sequence is that it can more effectively reflect its overall sequenceorder characteristics than the conventional correlation factors. Using such a formulation frame to represent the protein sequences means that the random forest algorithm can be used to conduct the prediction. The results for a large-scale independent test dataset show that the proposed model can achieve an excellent performance with an accuracy value
\end{abstract}


of about 0.86 and a geometry mean value of about 0.85 . The model is therefore a useful supplementary tool for PPI predictions. The predictor used in this article is freely available at http://www.jci-bioinfo.cn/PPI.

Key words: Chaos game representation; Wavelet transforms; Random forests; Protein-protein interactions

\section{INTRODUCTION}

Proteins play an important role in cell functions, such as cellular structure composition and promoting chemical reactions (Chen and Liu, 2005). The major functions of proteins are supported by the interactions between different proteins (Alberts et al., 2013). Over the past few years, the creation of a vast amount of protein data and associated data has meant that the interactions between proteins can be investigated in detail. Experimental determination of protein-protein interactions (PPIs) is currently mainly accomplished by the following three approaches: yeast two-hybrid assay (Ito et al., 2001), protein chips (Zhu et al., 2001), and mass spectrometry of purified protein complexes (Ho et al., 2002). However, these chemical or biological experimental methods are expensive, time-consuming, and labor-intensive (Han et al., 2005). The PPI information derived from statistical prediction can be complementary to the above biochemical methods. Furthermore, it can help to screen candidates for drug discovery, expedite the annotation of gene products, and provide insights into selecting relevant proteins for further study. To address the challenge, there is a need to develop reliable computational methods to facilitate the identification of PPIs based on non-structure sequence information, since structure information is hard to obtain (Bock and Gough, 2001; Guo et al., 2008).

Considerable efforts have been made to develop various statistical approaches over the past few years (Bock and Gough, 2001; Han et al., 2005; Ben-Hur and Noble, 2006; Chou and Cai, 2006). Some methods use genomic information, such as phylogenetic profiles (Pellegrini et al., 1999), gene neighborhood information (Overbeek et al., 1999), and gene fusion events (Marcotte et al., 1999). Protein structural information and sequence conservation between interacting proteins have also been used (Aloy and Russell, 2003; Ogmen et al., 2005; Planas-Iglesias et al., 2013a,b). Some approaches based on previously predicted (known protein) domains that are responsible for the interactions have also been proposed (Kim et al., 2002; Han et al., 2004; Morrison et al., 2006; Singhal and Resat, 2007). However, all the above methods cannot be implemented if the basic information about the proteins (especially some structure information) is not available.

If a sequence specifies the structure, then information about the amino acids in a protein might be sufficient to predict the interaction between two proteins for a specific biological function (Anfinsen, 1973). Several prediction methods based on sequence information have shown that the sequence information on amino acids alone may be sufficient to identify novel PPIs. Such an approach is universal. However, to date, it has been difficult to identify the most efficient protein sequence features for expressing a protein for PPIs prediction. Boch and Gough (2001) proposed an algorithm to predict PPIs by using several physiochemical descriptors. Nanni (2005a) also constructed an ensemble system to predict PPIs. However, these techniques are not robust since the local environments of the residues in the sequence are not considered. Shen et al. (2007) have developed a prediction method that performs well because the approach accounts for the properties of one amino acid and two vicinal ones. However, 
interactions usually occur in the discontinuous amino acid segments of the sequence and these relationships should also be considered when attempting to improve prediction accuracy. Furthermore, the prediction models used in these methods were developed using a limited number of training samples (often $<3000$ protein pairs), but with hundreds of variants. Therefore, there is an overfitting problem and the results are data-dependent.

In this paper, a machine learning method based on the random forest algorithm (Breiman, 2001) and chaos game representation (CGR) (Jeffrey, 1990; Fiser et al., 1994), combined with the wavelet transform (WT), was developed to predict PPIs using the primary protein sequences only. More than 10,000 PPIs were used to train the prediction model to avoid the problem of overfitting. The model encodes the order information for the protein using CGR and includes neighboring effects in the CGR plot. The CGR plot was then translated into numerical feature vectors by the WT process. Finally, the random forest algorithm was used to predict PPIs. This study has demonstrated that the model characterized the PPI information efficiently. The proposed model was tested on more than 30,000 independent protein pairs and yielded a good geometry mean (GM) result.

\section{MATERIAL AND METHODS}

\section{Data collection and data set construction}

To develop a PPI prediction model, we needed to construct or select a valid benchmark dataset to train and test the predictor. The PPI data used in this paper was collected from the publicly available Saccharomyces cerevisiae and Helicobacter pylori datasets.

In our experiments, $S$. cerevisiae (yeast) dataset was analyzed first. The data were downloaded from DIP (Database of Interacting Proteins, version 20140703) (Salwinski et al., 2004), which contained 22,775 positive pairs. The protein pairs that contained a protein with less than 50 amino acids were removed and then a non-redundant subset was generated at a sequence identity level of $40 \%$ by clustering analysis using the CD-HIT program ( $\mathrm{Li}$ and Godzik, 2006). The total positive dataset was reduced to 17,505 after these preprocessing procedures.

Non-interacting pairs were not readily available from the DIP, so we constructed them using the following methods. The negative dataset was generated based on an assumption that proteins located in different subcellular localizations do not interact. The subcellular location information for the proteins was extracted from Swiss-Prot (http://www.expasy.org/sprot/) and the positive data were localized to the cytoplasm, nucleus, mitochondria, endoplasmic reticulum, Golgi apparatus, peroxisome, and the vacuole. The negative data were obtained by pairing proteins from one location with proteins from other locations. The strategies had to meet the following requirements: i) the non-interacting pairs cannot appear in the positive dataset and ii) the contribution of proteins in the negative set should be as harmonious as possible. A total of 5943 negative pairs were generated via this approach.

However, Ben-Hur and Noble (2006) pointed out that restricting negative examples to different sub-location pairs leads to a biased estimate of the accuracy of a PPI predictor. So it is necessary to generate negative pairs with the same localization to reduce the effects of the bias. The protein pairs at the same localization were considered as negative pairs if they had not occurred in the yeast-positive pairs. From the seven sub-localizations, 27,204 negative pairs were generated ( 8000 from the cytoplasm, 8000 from the nucleus, 8284 from 
mitochondria, 1953 from the endoplasmic reticulum, 300 from the Golgi apparatus, 171 from peroxisomes, and 496 from the vacuole).

The H. pylori dataset comprised of 2916 protein pairs (1458 positive pairs and 1458 negative pairs) as described by Martin et al. (2005). This dataset was used to test the parameter sensitivities of the model and allowed us to compare our predictor with other predictors that have been used in the past.

\section{Protein representation}

One important step when predicting PPIs using sequence information is to formulate the protein sequences with an effective mathematical expression that can truly reflect the intrinsic correlations with their PPIs. To avoid completely losing the sequence-order information, the pseudo-amino acid composition (PseAAC) (Chou, 2001) was used to represent the protein sample. According to a recent review article (Chou, 2011), the general form of a PseAAC for a protein $\mathrm{P}$ is formulated by

$$
\mathrm{P}=\left[\begin{array}{llllll}
\sigma_{1} & \sigma_{2} & \cdots & \sigma_{u} & \cdots & \sigma_{\tau}
\end{array}\right] \quad \text { (Equation 1) }
$$

where the subscript $\tau$ is an integer, and its value as well as the component $\sigma_{1}, \sigma_{2}, \cdots$ will depend on how to extract the desired information form the amino acid sequence of P. Below, let us describe how to extract the core and essential features from a protein sequence to define the components in Equation 1.

\section{Encoding amino acids}

It is well-known that there are 20 amino acid types. The alphabet representation is very difficult to recognize, it is hard to compare different sequences, and one cannot use information from these abstract strings directly. Using graphical approaches to study biological problems can provide an intuitive picture or useful insights that help in the analysis of the complicated relationships in PPI systems. In order to discriminate patterns of protein sequences belonging to different functional classes, Fiser et al. (1994) proposed that CGR should be used to represent nucleotide sequences. In order to use CGR in PPI prediction, the protein needs to be transformed into nucleotide sequences. Many possible nucleotide sequences exist for one given amino acid sequence. In this study, the encoding method, shown in Table 1, proposed by Deschavanne and Tuffery (2008), was used because the encoding method keeps a balanced base composition, which maximizes the differences between the amino acid codes.

\begin{tabular}{|c|c|c|c|c|c|c|c|c|c|}
\hline \multicolumn{10}{|c|}{ En 1} \\
\hline $\mathrm{A}=\mathrm{GCT}$ & $\mathrm{G}=\mathrm{GGT}$ & $\mathrm{M}=\mathrm{ATG}$ & $\mathrm{S}=\mathrm{TCA}$ & $\mathrm{C}=\mathrm{TGC}$ & $\mathrm{H}=\mathrm{CAC}$ & $\mathrm{N}=\mathrm{AAC}$ & $\mathrm{T}=\mathrm{ACT}$ & $\mathrm{D}=\mathrm{GAC}$ & $\mathrm{I}=\mathrm{ATT}$ \\
\hline $\mathrm{P}=\mathrm{CCA}$ & $\mathrm{V}=\mathrm{GTG}$ & $\mathrm{E}=\mathrm{GAG}$ & $\mathrm{K}=\mathrm{AAG}$ & $\mathrm{Q}=\mathrm{CAG}$ & $\mathrm{W}=\mathrm{TGG}$ & $\mathrm{F}=\mathrm{TTC}$ & $\mathrm{L}=\mathrm{CTA}$ & $\mathrm{R}=\mathrm{CGA}$ & $\mathrm{Y}=\mathrm{TAC}$ \\
\hline
\end{tabular}

The encoding approach was according to Deschavanne and Tuffery (2008). 


\section{CGR and two related discrete series}

Table 1 shows how a protein sequence can be transformed into a nucleotide sequence, which means that a CGR-plot for nucleotide sequences can be used to analyze it. A brief outline of the CGR procedure is:

In a $[0,1] \times[0,1]$ square, the four vertices of the defined square correspond to the four letters: A, C, G, and T. Then, the CGR-plot can be obtained via the following steps: 1) put an initial point at the center of the square; 2) place the second point at the half way between the initial point and the vertex corresponding to the first letter of the nucleotide sequence; 3 ) place the $i^{\text {th }}$ point half way between the $(i-1)^{\text {th }}$ point and the vertex corresponding to the $i^{\text {th }}$ letter; 4$)$ go to Step 3 until you reach the end of the nucleotide sequence.

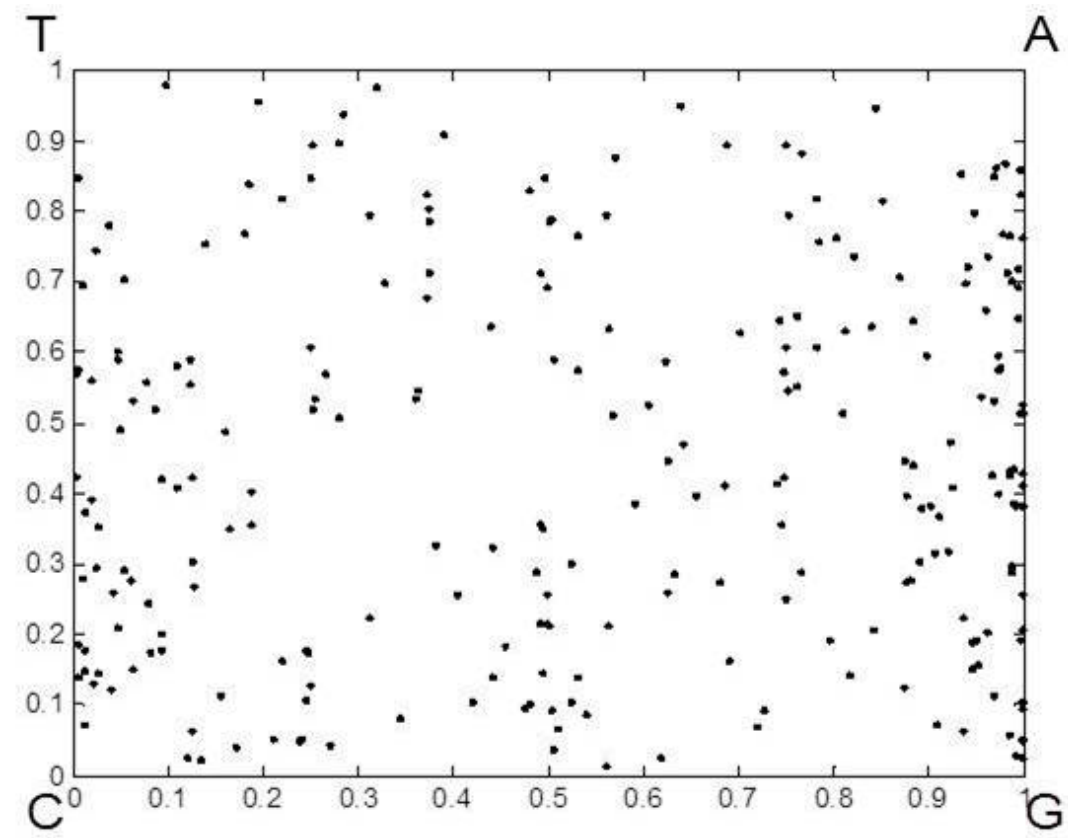

Figure 1. CGR-plot of a protein sequence. One point in the plot represents one corresponding nucleotide in the converted protein sequence. The order for the points (corresponding to the order in the converted protein sequence) is saved. Details about the plotting procedure can be found in the text.

Using these steps enabled us to obtain a CGR-plot of the nucleotide sequence. One point in the CGR represents one nucleotide. The CGR-plot of a typical protein is shown in Figure 1 as an example. Many important features, which, at the beginning, are hidden in a long and complicated nucleotide sequence, can be clearly revealed via its CGR. However, it is difficult to analyze the CGR-plot directly. In this study, we divided the CGR-plot into two related discrete series because any point in the CGR-plot is determined by the $\mathrm{x}$ and $\mathrm{y}$ coordinates. We regarded the $\mathrm{x}$ and $\mathrm{y}$ coordinates as two digital signals, and therefore two discrete series related to the CGR-plot can be extracted. Figure 2 shows the two series for the CGR-plot shown in Figure 1. We denote them as CGRx and CGRy, respectively. 
a

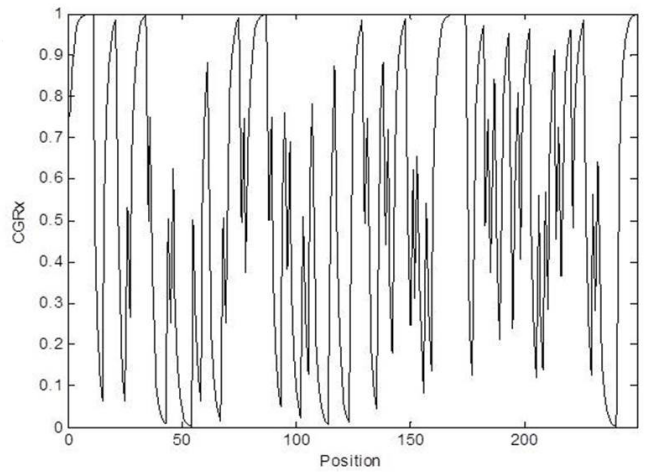

b

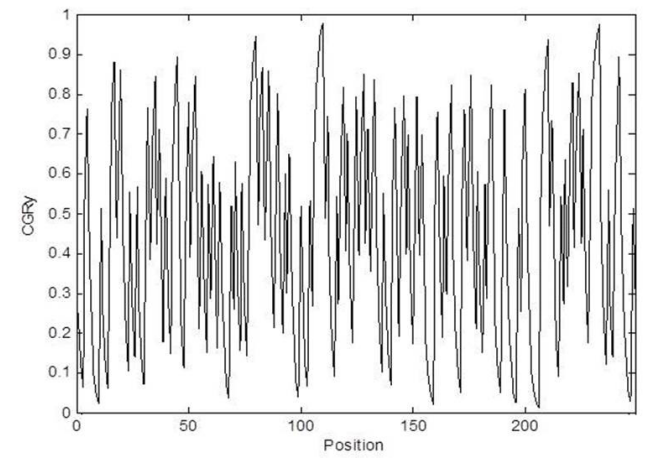

Figure 2. Time series related to Figure 1. The first time series (shown in a) is used to represent the x-coordinate of the points in Figure 1 and the second (shown in $\mathbf{b}$ ) is used to represent the y-coordinate of the points in Figure 1.

It has been proven that a CGR-plot can provide useful information. Because two discrete series are obtained from the CGR-plot, all the information stored in the CGR-plot is contained in these two series. Therefore, an indirect analysis of the protein primary sequence is possible through analysis of the two series.

\section{Discrete wavelet transform (DWT)}

The WT process (Mallat, 1989; Daubechies, 1990) is a multi-resolution analysis tool. It has been used to analyze de-noising, and to compress signals and images (Devore et al., 1992). WT decomposes a signal into a set of basic functions. These basic functions are called wavelets. Wavelets are obtained from a single-prototype wavelet called the mother wavelet by dilations and shifting:

$$
\Psi_{a, b}(t)=\frac{1}{\sqrt{a}} \Psi\left(\frac{t-b}{a}\right)
$$

(Equation 2)

where $\Psi(\mathrm{t})$ is the mother wavelet, $a$ is the scaling parameter and $b$ is the shifting parameter. The 1-D wavelet transform is given by: 


$$
W_{f}(a, b)=\int_{-\infty}^{\infty} x(t) \Psi_{a, b}(t) d t
$$

(Equation 3)

where $x(t)$ is the decomposed signal. The DWT changes a discrete time signal into a discrete wavelet representation. It converts an input series into one high-pass wavelet coefficient series and one low-pass wavelet coefficient series (half the length of the input series).

In practice, such a transform will be applied recursively on the low-pass series using the Mallat algorithm (Mallat, 1999) until the desired number of iterations is reached. The following block diagram (see Figure 3) depicts the digital implementation of DWT.

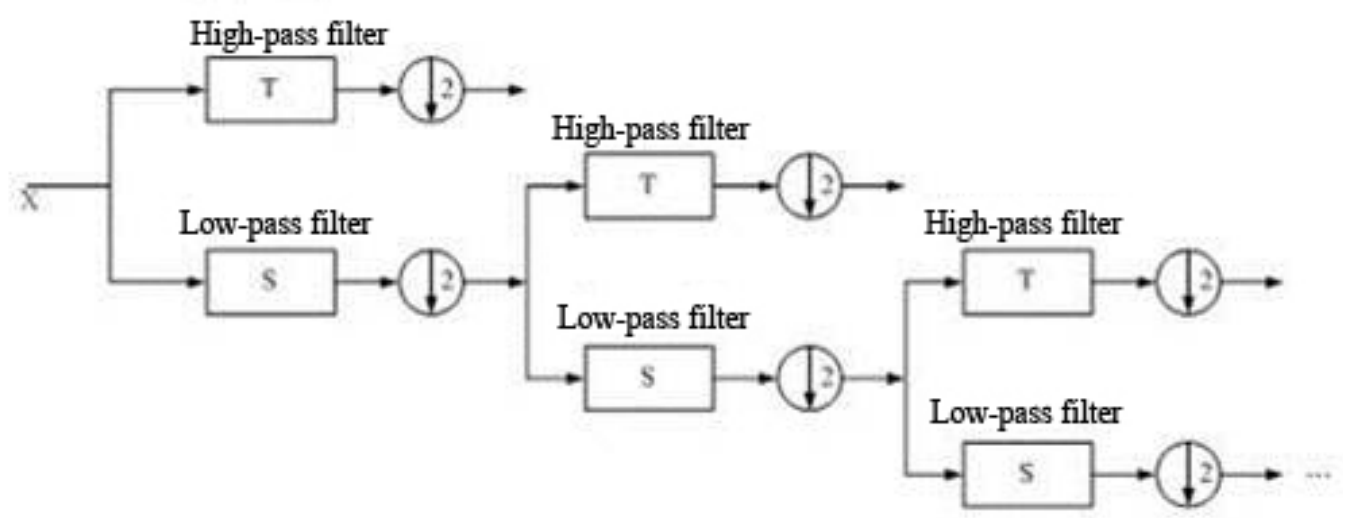

Figure 3. Multi-level DWT procedure.

In this paper, the two discrete series, CGRx and CGRy, were dealt with by 5-level discrete wavelet decomposition (The undersample procedure exits in DWT meant that the decomposition level could not be too large). This allowed us to obtain six sub-bands. For each sub-band, we extracted the mean value, and the maximum, minimum and standard deviations as the features. This resulted in a 48 -dimensional vector for each protein $(6 \times 4 \times 2=48$, where 6 is the number of sub-bands, 4 is the number of descriptors for each sub-band, and 2 is the number of discrete series for each protein, i.e., CGRx and CGRy). Therefore, a 96-dimensional vector can be used to represent a protein pair.

\section{Random forest algorithm}

The random forest algorithm (Breiman, 2001) is an ensemble of unpruned classification and regression trees that operates by constructing a multitude of decision trees during the training period and outputting the final class that is the majority vote of the classes output by individual trees. These trees are generated by the bootstrap procedure for the training data and by using random-feature selection in the tree generation process. The random forest algorithm usually produces a remarkable improvement in performance over the single-decision tree classifier, such as CART and C4.5 (Mitchell, 1997), which are often used as the base learner in the random forest algorithm. Furthermore, the random forest algorithm shows a good generaliza- 
tion error rate when compared to Adaboost, and is more robust to noise. In our experiments, we found that the random forest algorithm was not sensitive to the number of trees when it was used in our model. A total of 200 trees were used in order to alleviate computational cost and the overfitting problem. A schematic diagram of the proposed method is shown in Figure 4.

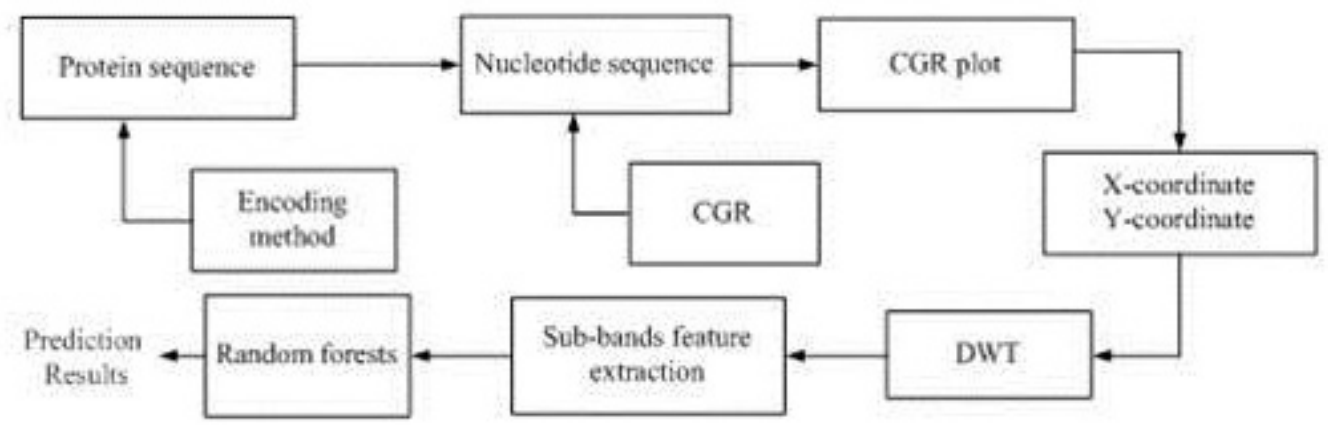

Figure 4. Proposed framework for the WT CGR.

\section{Web server and user guide}

To enhance the value of its practical application, a web-server for PPI predictors was established at the web-site http://www.jci-bioinfo.cn/PPI. Moreover, for convenience, a step-by-step guide is provided on how to use the web server predictors. A summary of the guide is given below:

1) go to the internet at http://www.jci-bioinfo.cn/PPI where you will see the top page for the predictor on your computer screen, as shown in Figure 5.

PPI: Prediction of Protein-Protein Interactions with Chaos Game Representation and Wavelets Transform via Random Forests

\section{| Read Me | Supporting Information | Citation |}

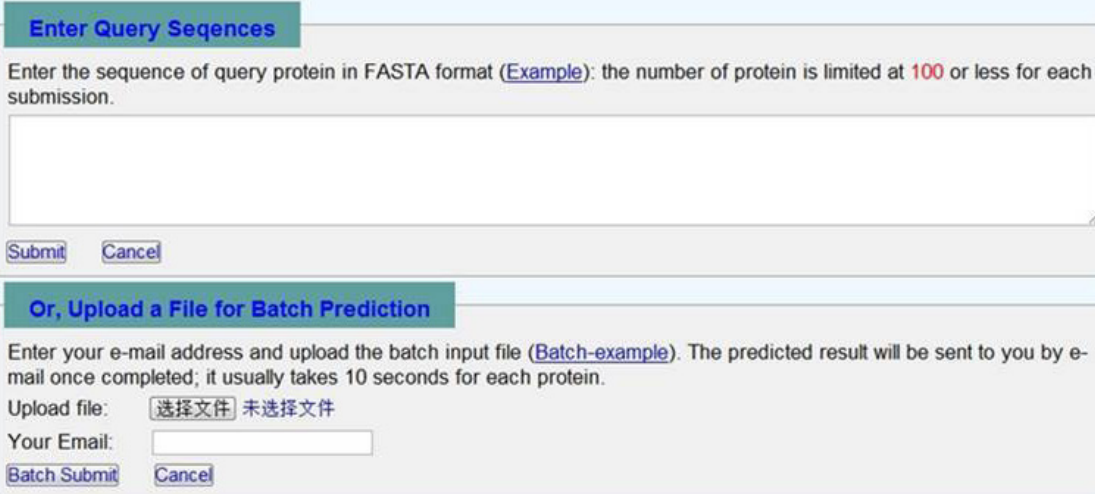

Contact @ idzxiaoxuan@163.com

Figure 5. Screenshot of the PPI predictor webpage. 
Click on the Read Me button to see a brief introduction about the PPI predictor; 2) when the predicted PPIs are found in several protein sequences, you can either type or copy/paste the query protein sequence into the input box at the top half of Figure 5. It is important to note that the input sequence should be in the FASTA format. A sequence in FASTA format consists of a single-initial line beginning with a greater-than symbol $(>)$ in the first column, followed by lines of sequence data. The words right after the ">" symbol in the single-initial line are optional and are only used for identification and description purposes; 3 ) to get the predicted result, you only need to click on the Submit button. For example, if you use the query amino acid sequences in the Example window as the input, you will see the status of your job on the screen. When the job is complete, the result will be displayed on the page. Jobs can be accomplished within $15 \mathrm{~s}$ in most cases. However, the length of sequence is the key time-consuming factor: the longer the query protein sequence is the more time is usually needed; 4) as shown in the lower panel of Figure 5, you may also choose batch prediction by entering your e-mail address and your desired batch input file (in FASTA format) via the "Browse" button. To see an example of a batch input file, click on the Batch-example button; 5) clicking the Citation button, will show the relevant papers that document the detailed development of the predictor; 6) clicking on the Supporting Information button will download the benchmark dataset used to train and test the PPI predictor.

\section{RESULTS AND DISCUSSION}

The literature suggests that six metrics are often used to score the quality of a predictor at six different angles. These include accuracy (Acc), sensitivity (Sen), specificity (Spec), F-measure (Fm), Mathew correlation coefficient (MCC), and GM. These metrics are defined in Table 2. Sen and Spec illustrate the correct prediction ratios for positive and negative data sets, respectively. The overall accuracy is measured as the average of Sen and Spec. However, when the numbers of positive data and negative data differ too much from each other, the MCC should be calculated to assess the prediction performance. The MCC value ranges from -1 to 1 , and a larger MCC equates to better prediction performance.

Table 2. Evaluation measurements.

\begin{tabular}{lll}
\hline Measurement & Abbreviation & Equation \\
\hline Accuracy & Acc & $(\mathrm{TP}+\mathrm{TN}) /(\mathrm{TP}+\mathrm{TN}+\mathrm{FP}+\mathrm{FN})$ \\
Sensitivity & $\mathrm{Sen}$ & $\mathrm{TP} /(\mathrm{TP}+\mathrm{FN})$ \\
Specificity & $\mathrm{Spec}$ & $\mathrm{TN} /(\mathrm{TN}+\mathrm{FN})$ \\
F-measure & $\mathrm{Fm}$ & $2 \mathrm{TP} /(2 \mathrm{TP}+\mathrm{FP}+\mathrm{FN})$ \\
Mathew correlation coefficient & $\mathrm{MCC}$ & $\frac{\mathrm{TP} * \mathrm{TN}-\mathrm{FN} * \mathrm{FP}}{\sqrt{(\mathrm{TP}+\mathrm{FN}) *(\mathrm{TN}+\mathrm{FP}) *(\mathrm{TP}+\mathrm{FP}) *(\mathrm{TN}+\mathrm{FN})}}$ \\
& & $\mathrm{sqrt}((\mathrm{TP} /(\mathrm{TP}+\mathrm{FP})) \times(\mathrm{TN} /(\mathrm{TN}+\mathrm{FN})))$ \\
\hline
\end{tabular}

TP: number of true positives; FP: number of false positives; TN: number of true negatives; FN: number of false negatives.

After a set of clear and valid metrics has been defined to measure the quality of a predictor (Table 2), it is then necessary to consider how to objectively derive the values of these metrics for a predictor. In statistical prediction, the following three cross-validation methods are often used to calculate the metrics shown in Table 2: the independent dataset test, the sub- 
sampling (e.g., 2-, 5-, or 10-fold cross-validation) test, and the jackknife test. The jackknife test is thought to be the least arbitrary because it can always yield a unique result for a given benchmark dataset. Therefore, the jackknife test has been increasingly and widely adopted to test the power of various prediction methods. However, to reduce computational time, we adopted the 5- or 10-fold cross-validation tests in this study, which have been adopted by many investigators when the random forest algorithm is used as the prediction engine. In $K$-fold cross-validation, the original sample is randomly partitioned into $K$ subsamples. A single subsample is retained as the validation data for testing the model, and the remaining subsamples are used as training data. The cross-validation process is then repeated $K$ times (the folds), with each of the $K$ subsamples used exactly once as the validation data. The advantage of this method is that all observations are used for both training and validation, which gives it a high computational efficiency; thus, it has been used to check many protein attribute prediction models. However, as can be seen, the partition is random, so the results are often variable.

\section{Effect of wavelet functions}

The H. pylori datasets were used to evaluate the effect of the wavelet functions. The influence of the wavelet functions were analyzed because a suitable wavelet basis can match the underlying structure of the signal better and a more reliable feature can be extracted from the original protein sequences. Some properties of the wavelet basis, such as compact support, orthogonality, symmetry, smoothness, and order of vanishing moments, must be considered during signal processing. It was hoped that our wavelet functions would have these aforementioned properties. However, there were many conflicting conditions that restricted the selection of them. For example, none of wavelet basis functions had all these desirable properties simultaneously. In recent decades, Daubechies (1990) constructed a class of orthonormal wavelet basis functions with compact support and smooth properties. In this study, five Daubechies wavelet functions were tested: Daubechies number 1 (DB1), Daubechies number 2 (DB2), Daubechies number 3 (DB3), Daubechies number 4 (DB4), and Daubechies number 5 (DB5). Table 3 shows that the training accuracy reached 0.8570 when using the random forest algorithm as the classifier when the DB1 wavelet function was used to extract the features. The number of random forest trees was 200 and the number of mtrys (dimension of subspace for constructing the decision trees) was 20 . However, when other wavelet functions were used, the training Acc ranged from 0.8356 to 0.8475 . Moreover, other performance measures, such as GM, Sen, Spec, Fm, and MCC, were investigated. Table 3 also shows that DB1 was the best function. These results may have been caused by the fact that the DB1 wavelet possesses a lower vanish moment. Increased numbers of non-zero coefficients generated after decomposition and the diverse trees used in the random forest algorithm were easy obtained because the diversity of component learners required. For a single learner, a higher vanish moment wavelet function, such as DB4 is needed. In this study, the DB1 wavelet function was selected as the appropriate wavelet function in our experiments.

\section{Performance using the $S$. cerevisiae dataset}

The proposed predictor was first applied to the $S$. cerevisiae dataset. The dataset consisted of 17,505 positive pairs and 33,147 negative pairs. A total of 5943 positive pairs and 5943 negative pairs were randomly selected from $S$. cerevisiae as the training dataset. 
Table 3. Performance of the different wavelet functions on the Helicobacter pylori dataset by 10 cross-validations

\begin{tabular}{|c|c|c|c|c|c|c|}
\hline \multirow[t]{2}{*}{ Wavelet functions } & \multicolumn{6}{|c|}{ Evaluation methods } \\
\hline & Accuracy & F-measure & Geometry mean & Sensitivity & Specificity & MCC \\
\hline$\overline{\mathrm{DB} 1}$ & 0.8570 & 0.8576 & 0.8569 & 0.8509 & 0.8646 & 0.7151 \\
\hline DB2 & 0.8474 & 0.8491 & 0.8473 & 0.8387 & 0.8568 & 0.6954 \\
\hline DB3 & 0.8462 & 0.8481 & 0.8459 & 0.8363 & 0.8576 & 0.6933 \\
\hline DB4 & 0.8356 & 0.8374 & 0.8352 & 0.8276 & 0.8448 & 0.6720 \\
\hline DB5 & 0.8375 & 0.8388 & 0.8375 & 0.8308 & 0.8445 & 0.6854 \\
\hline
\end{tabular}

The remaining pairs were used as the independent test dataset. A 5-fold cross-validation was used to evaluate the predictor for the training dataset, and the procedure was repeated 10 times. The results for the training dataset are shown in Table 4.

Table 4. Five-fold cross-validation results for the training data (Saccharomyces cerevisiae dataset).

\begin{tabular}{|c|c|c|c|c|c|c|}
\hline & \multicolumn{6}{|c|}{ Evaluation methods } \\
\hline & Accuracy & Sensitivity & Specificity & F-measure & $\mathrm{MCC}$ & Geometry mean \\
\hline 1 & $0.8348 \pm 0.0026$ & $0.8655 \pm 0.0064$ & $0.8091 \pm 0.0078$ & $0.8276 \pm 0.0032$ & $0.6721 \pm 0.0047$ & $0.8337 \pm 0.0025$ \\
\hline 2 & $0.8326 \pm 0.0023$ & $0.8621 \pm 0.0105$ & $0.8075 \pm 0.0104$ & $0.8254 \pm 0.0025$ & $0.6675 \pm 0.0045$ & $0.8316 \pm 0.0021$ \\
\hline 3 & $0.8418 \pm 0.0055$ & $0.8711 \pm 0.0143$ & $0.8169 \pm 0.0034$ & $0.8353 \pm 0.0070$ & $0.6859 \pm 0.0122$ & $0.8410 \pm 0.0059$ \\
\hline 4 & $0.8414 \pm 0.0038$ & $0.8663 \pm 0.0073$ & $0.8196 \pm 0.0069$ & $0.8357 \pm 0.0061$ & $0.6844 \pm 0.0076$ & $0.8407 \pm 0.0043$ \\
\hline 5 & $0.8411 \pm 0.0070$ & $0.8711 \pm 0.0143$ & $0.8157 \pm 0.0030$ & $0.8345 \pm 0.0054$ & $0.6844 \pm 0.0149$ & $0.8400 \pm 0.0064$ \\
\hline 6 & $0.8344 \pm 0.0062$ & $0.8612 \pm 0.0162$ & $0.8115 \pm 0.0037$ & $0.8281 \pm 0.0064$ & $0.6709 \pm 0.0135$ & $0.8336 \pm 0.0061$ \\
\hline 7 & $0.8304 \pm 0.0039$ & $0.8536 \pm 0.0124$ & $0.8105 \pm 0.0095$ & $0.8246 \pm 0.0056$ & $0.6624 \pm 0.0077$ & $0.8296 \pm 0.0041$ \\
\hline 8 & $0.8374 \pm 0.0045$ & $0.8602 \pm 0.0072$ & $0.8173 \pm 0.0078$ & $0.8321 \pm 0.0036$ & $0.6761 \pm 0.0088$ & $0.8367 \pm 0.0042$ \\
\hline 9 & $0.8369 \pm 0.0082$ & $0.8628 \pm 0.0115$ & $0.8143 \pm 0.0139$ & $0.8308 \pm 0.0083$ & $0.6755 \pm 0.0162$ & $0.8361 \pm 0.0081$ \\
\hline 10 & $0.8310 \pm 0.0105$ & $0.8580 \pm 0.0061$ & $0.8083 \pm 0.0223$ & $0.8243 \pm 0.0119$ & $0.6643 \pm 0.0194$ & $0.8302 \pm 0.0109$ \\
\hline Mean & $0.8362 \pm 0.0040$ & $0.8632 \pm 0.0055$ & $0.8131 \pm 0.0043$ & $0.8298 \pm 0.0044$ & $0.6744 \pm 0.0085$ & $0.8353 \pm 0.0043$ \\
\hline Guo & $0.7796 \pm 0.0031$ & $0.7684 \pm 0.0031$ & $0.7822 \pm 0.0043$ & $0.7864 \pm 0.0035$ & $0.5099 \pm 0.0062$ & $0.7791 \pm 0.0031$ \\
\hline
\end{tabular}

Table 4 shows that the proposed model performed well with the training dataset. The average results produced by the model were 0.8362 for Acc, 0.6744 for MCC, 0.8353 for GM, 0.8298 for Fm, 0.8632 for Sen, and 0.8131 for Spec.

After the 5-fold cross-validation, the remaining pairs in the independent test dataset were applied to further evaluate the proposed predictors. A total of 11,562 positive samples and 21,261 negative samples were included in the test data. The experimental results are shown in Table 5.

Table 5. Five-fold cross-validation results for the independent test data (Saccharomyces cerevisiae dataset).

\begin{tabular}{|c|c|c|c|c|c|c|}
\hline & \multicolumn{6}{|c|}{ Evaluation methods } \\
\hline & Accuracy & Sensitivity & Specificity & F-measure & $\mathrm{MCC}$ & Geometry mean \\
\hline 1 & 0.8584 & 0.7917 & 0.8961 & 0.8015 & 0.6916 & 0.8470 \\
\hline 2 & 0.8639 & 0.8046 & 0.8965 & 0.8075 & 0.7022 & 0.8507 \\
\hline 3 & 0.8628 & 0.8035 & 0.8954 & 0.8058 & 0.6998 & 0.8493 \\
\hline 4 & 0.8597 & 0.7959 & 0.8953 & 0.8025 & 0.6938 & 0.8473 \\
\hline 5 & 0.8617 & 0.8008 & 0.8954 & 0.8047 & 0.6977 & 0.8486 \\
\hline 6 & 0.8595 & 0.7886 & 0.9006 & 0.8047 & 0.6954 & 0.8503 \\
\hline 7 & 0.8596 & 0.7896 & 0.8999 & 0.8044 & 0.6952 & 0.8499 \\
\hline 8 & 0.8603 & 0.7945 & 0.8973 & 0.8040 & 0.6956 & 0.8489 \\
\hline 9 & 0.8569 & 0.7879 & 0.8962 & 0.8000 & 0.6888 & 0.8461 \\
\hline 10 & 0.8667 & 0.8016 & 0.9038 & 0.8136 & 0.7101 & 0.8569 \\
\hline Mean & $0.8610 \pm 0.0027$ & $0.7959 \pm 0.0060$ & $0.8977 \pm 0.0027$ & $0.8049 \pm 0.0036$ & $0.6970 \pm 0.0057$ & $0.8495 \pm 0.0030$ \\
\hline Guo & $0.7865 \pm 0.0030$ & $0.6485 \pm 0.0044$ & $0.8500 \pm 0.0034$ & $0.7219 \pm 0.0029$ & $0.5171 \pm 0.0048$ & $0.7929 \pm 0.0025$ \\
\hline
\end{tabular}


Table 5 shows that the proposed model performed well with the test dataset. The average results of the predictor for the test dataset were 0.8610 for Acc, 0.6970 for MCC, 0.8495 for GM, 0.8049 for Fm, 0.7959 for Sen, and 0.8977 for Spec.

\section{Comparison with other methods}

We compared the effectiveness of our proposed model with the method proposed by Guo et al. (2008). Their model also only used the sequence features to predict the PPIs. The auto covariance (AC) features and support vector machine (SVM) were used for prediction. AC accounts for the interactions between residues at a certain distance apart in the sequence, so this model mainly takes the neighborhood effect into account. The definition of $\mathrm{AC}$ is as follows:

$$
A C_{\text {lag, }, j}=\frac{1}{n-\operatorname{lag}} \sum_{i=1}^{n-l a g}\left(X_{i, j}-\frac{1}{n} \sum_{i=1}^{n} X_{i, j}\right) \times\left(X_{(i+l a g), j}-\frac{1}{n} \sum_{i=1}^{n} X_{i, j}\right)
$$

(Equation 4)

where $j$ represents a descriptor, such as the physiochemical descriptors of the amino acids that make up the protein, $i$ is the position in sequence $X, n$ is the length of sequence $X$, and lag is the value of the lag (the maximum distance between an amino acid residue and a neighbor that is a certain number of residues away). In our study, when we used the $\mathrm{AC}$ model, a protein pair was converted into a 420 -dimensional $(2 \times 30 \times 7)$ vector with a lag of 30 amino acids, where 2 is the number of two protein sequences and 7 is the number of descriptors. The experimental results for the training and testing data are shown in Figure 6.

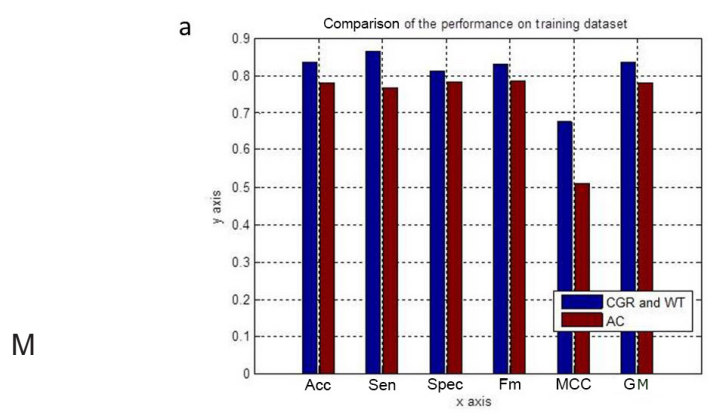

b

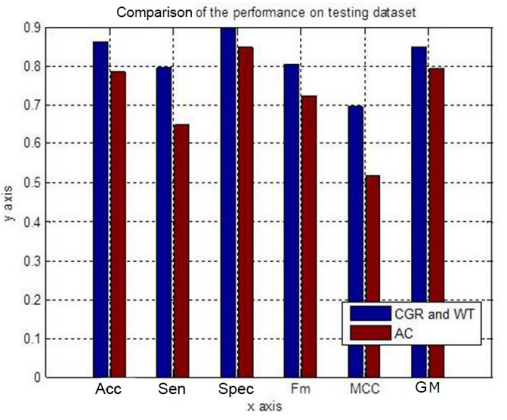

Figure 6. Comparison between the proposed predictor and the Guo et al. (2008) predictor for a. the training dataset and $\mathbf{b}$. the testing dataset. The value shown in the figure is the mean value of 10 runs. 
Figure 6 shows that the performance of the proposed model was better than that of the AC model, especially for the MCC value. This means that the proposed model is more accurate for both negative and positive samples, and has a better prediction ability. Furthermore, the number of extracted features used in our model was only 96, which was less than the 420 features used by the AC model. Furthermore, the AC model used 5 cross-validations to select the SVM's parameters, which is complex and tedious. Therefore, we used less features to reduce computational cost, but obtained a better performance.

We compared the results of the proposed method with the existing methods using the H. pylori dataset. The results of the 10-fold cross-validation for four different methods (Bock and Gough, 2003; Martin et al., 2005; Nanni, 2005a,b) for the H. pylori dataset are shown in Table 6. In Bock and Gough's approach (Bock and Gough, 2001, 2003), several structural and physiochemical descriptors, with SVM as the classifier, were used to predict PPIs. Martin et al. (2005) developed a novel descriptor called a signature product, which uses an expansion of the signature descriptor from chemical informatics to infer PPIs. Nanni (2005a) developed a PPI predictor based on the K-local hyperplane. In another paper, Nanni (2005b) proposed a PPIs predictor based on linear discriminant classifiers.

Table 6. Comparison between different methods using 10 cross validations.
\begin{tabular}{lccc}
\hline Methods & Sensitivity & Precision & Accuracy \\
\hline Bock and Gough $^{\mathrm{a}}$ & 0.6980 & 0.8020 & 0.7580 \\
Martin $^{\mathrm{b}}$ & 0.7990 & 0.8570 & 0.8340 \\
Nannic $^{\mathrm{c}}$ & 0.8060 & 0.8510 & 0.8300 \\
Nanni $^{\mathrm{d}}$ & 0.8600 & 0.8400 & 0.8400 \\
Our method $^{\mathrm{e}}$ & 0.8510 & 0.8760 & 0.8570 \\
\hline
\end{tabular}

Prec $=\mathrm{TP} /(\mathrm{TP}+\mathrm{FP})$ a Results obtained by the 10 cross-validations for the predictor by Bock and Gough (2003) for the H. pylori dataset. See the "CGR and two related discrete series" section for further explanation about 10 crossvalidations. ${ }^{b}$ Results obtained by the 10 cross-validations for the predictor by Martin et al. (2005) for the H. pylori dataset. ${ }^{~} R$ Results obtained by the 10 cross-validations for the predictor by Nanni (2005a) for the $H$. pylori dataset. ${ }^{\mathrm{d}}$ Results obtained by the 10 cross-validations for the predictor by Nanni (2005b) for the H. pylori dataset. ${ }^{\mathrm{e}}$ Results obtained by the 10 cross-validations for our predictor for the $H$. pylori dataset.

Our method clearly achieved better results than the other four approaches, especially for accuracy and precision. Only the sensitivity was slightly lower than one of the Nanni et al. (2005b) methods. The results on the two datasets showed that the proposed predictor was a useful supplementary tool for PPI prediction.

\section{CONCLUSIONS}

This study investigated a new PPI prediction model that only used the primary sequences of proteins. The protein features were extracted using the CGR and WT, and the random forest algorithm was used for prediction. We then evaluated the model on a large test dataset. The prediction results clearly showed that our model could effectively predict PPIs. Furthermore, fewer features were used in the model but a better performance was achieved. The PPI predictor is available at (http://www.jci-bioinfo.cn/PPI). 


\section{ACKNOWLEDGMENTS}

Research partially supported by the National Nature Science Foundation of China (Grants \#61261027, \#61262038, \#31260273, and \#61202313), the Natural Science Foundation of Jiangxi Province, China (Grants \#20122BAB211033, \#20122BAB201044, and \#20132BAB201053), the Scientific Research Plan of the Department of Education of Jiangxi Province (\#GJJ14640), and The Young Teacher Development Plan of Visiting Scholars Program in the University of Jiangxi Province (Grant \#2014). The funders had no role in study design, data collection and analysis, decision to publish, or preparation of the manuscript.

\section{REFERENCES}

Alberts B, Bray D, Hopkin K, Johnson A, et al. (2013). Essential cell biology. 3rd edn. Garland Science, New York.

Aloy P and Russell RB (2003). InterPreTS: protein interaction prediction through tertiary structure. Bioinformatics 19: 161-162.

Anfinsen CB (1973). Principles that govern the folding of protein chains. Science 181: 223-230.

Ben-Hur A and Noble WS (2006). Choosing negative examples for the prediction of protein-protein interactions. $B M C$ Bioinformatics 7: S2.

Bock JR and Gough DA (2001). Predicting protein-protein interactions from primary structure. Bioinformatics 17: 455460.

Bock JR and Gough DA (2003). Whole-proteome interaction mining. Bioinformatics 19: 125-134.

Breiman I (2001). Random forests. Mach. Learn. 45: 5-32.

Chen XW and Liu M (2005). Prediction of protein-protein interactions using random decision forest framework. Bioinformatics 21: 4394-4400.

Chou K (2001). Prediction of protein cellular attributes using pseudo amino acid composition. Proteins 43: 246-255.

Chou KC (2011). Some remarks on protein attribute prediction and pseudo amino acid composition. J. Theor. Biol. 273 : 236-247.

Chou KC and Cai YD (2006). Predicting protein-protein interactions from sequences in a hybridization space. J. Proteome Res. 5: 316-322.

Daubechies I (1990). The wavelet transform, time-frequency localization and signal analysis. IEEE Inform. Theory 36: 961-1005.

Deschavanne P and Tuffery P (2008). Exploring an alignment free approach for protein classification and structural class prediction. Biochimie 90: 615-625.

Devore RA, Jawerth B and Lucier BJ (1992). Image compression through wavelet transform coding. IEEE Inform. Theory 2: 719-746.

Fiser A, Tusnady GE and Simon I (1994). Chaos game representation of protein structures. J. Mol. Graphics 12: 302-304.

Guo Y, Yu L, Wen Z and Li ML, et al. (2008). Using support vector machine combined with auto covariance to predict protein-protein interactions from protein sequences. Nucleic Acids Res. 36: 3025-3030.

Han DS, Kim HS, Jang WH, Lee SD, et al. (2004). PreSPI: a domain combination based prediction system for proteinprotein interaction. Nucleic Acids Res. 32: 6312-6320.

Han JDJ, Dupuy D, Bertin N, Cusick ME, et al. (2005). Effect of sampling on topology predictions of protein-protein interaction networks. Nat. Biotechnol. 23: 839-844.

Ho Y, Gruhler A, Heilbut A, Bader GD, et al. (2002). Systematic identification of protein complexes in Saccharomyces cerevisiae by mass spectrometry. Nature 415: 180-183.

Ito T, Chiba T, Ozawa R, Yoshida M, et al. (2001). A comprehensive two-hybrid analysis to explore the yeast protein interactome. P. Natl. Acad. Sci. U. S. A. 98: 4569-4574.

Jeffrey HJ (1990). Chaos game representation of gene structure. Nucleic Acids Res. 18: 2163-2170.

Kim WK, Park J and Suh JK (2002). Large scale statistical prediction of protein-protein interaction by potentially interacting domain (PID) pair. Genome Inform. 13: 42-50.

Li W and Godzik A (2006). Cd-hit: a fast program for clustering and comparing large sets of protein or nucleotide sequences. Bioinformatics 22: 1658-1659.

Mallat S (1999). A wavelet tour of signal processing. 3rd edn. Academic Press, New York.

Mallat SG (1989). A theory for multiresolution signal decomposition: the wavelet representation. IEEE T. Pattern Anal. 
11: 674-693.

Marcotte EM, Pellegrini M, Ng HL, Rice DW, et al. (1999). Detecting protein function and protein-protein interactions from genome sequences. Science 285: 751-753.

Martin S, Roe D and Faulon JL (2005). Predicting protein-protein interactions using signature products. Bioinformatics 21: $218-226$.

Mitchell TM (1997). Machine learning. McGraw Hill, Burr Ridge.

Morrison JL, Breitling R, Higham DJ and David R Gilbert (2006). A lock-and-key model for protein-protein interactions. Bioinformatics 22: 2012-2019.

Nanni L (2005a). Fusion of classifiers for predicting protein-protein interactions. Neurocomputing 68: 289-296.

Nanni L (2005b). Hyperplanes for predicting protein-protein interactions. Neurocomputing 69: 257-263.

Ogmen U, Keskin O, Aytuna AS, Nussinov R, et al. (2005). PRISM: protein interactions by structural matching. Nucleic Acids Res. 33: W331-W336.

Overbeek R, Fonstein M, D'Souza M, Pusch GD, et al. (1999). Use of contiguity on the chromosome to predict functional coupling. In Silico Biol. 1: 93-108.

Pellegrini M, Marcotte EM, Thompson MJ, David Eisenberg, et al. (1999). Assigning protein functions by comparative genome analysis: protein phylogenetic profiles. P. Natl. Acad. Sci. USA. 96: 4285-4288.

Planas-Iglesias J, Bonet J, Garca-Garca J, Marín-López MA, et al. (2013a). Understanding protein-protein interactions using local structural features. J. Mol. Biol. 425: 1210-1224.

Planas-Iglesias J, Marin-Lopez MA, Bonet J, Garcia-Garcia J, et al. (2013b). iLoops: a protein-protein interaction prediction server based on structural features. Bioinformatics: btt401.

Salwinski L, Miller CS, Smith AJ, Pettit FK, et al. (2004). The database of interacting proteins. Nucleic Acids Res. 32: D449-D451.

Shen J, Zhang J, Luo X, Zhu W, et al. (2007). Predicting protein-protein interactions based only on sequences information. P. Natl. Acad. Sci. U. S. A. 104: 4337-4341.

Singhal M and Resat H (2007). A domain-based approach to predict protein-protein interactions. BMC Bioinformatics 8: 199.

Zhu H, Bilgin M, Bangham R, Hall D, et al. (2001). Global analysis of protein activities using proteome chips. Science 293: 2101-2105. 